\title{
Histomorphological Spectrum of Hepatic Lesions in Liver Biopsies - A Prospective Hospital Based Study
}

\author{
Authors \\ Shivani Gandhi ${ }^{1 *}$, Arvind Khajuria ${ }^{2}$, K.C. Goswami ${ }^{3}$ \\ ${ }^{1 *}$ Post Graduate, Department of Pathology, Acharya Shri Chander College of Medical Science \& Hospital, \\ Sidhra, Jammu (J\&K), India. \\ ${ }^{2}$ Professor \& Head of Department, Department of Pathology, Acharya Shri Chander College of Medical \\ Science \& Hospital, Sidhra, Jammu (J\&K), India. \\ ${ }^{3}$ Professor, Department of Pathology, Acharya Shri Chander College of Medical Science \& Hospital, Sidhra, \\ Jammu (J\&K), India \\ *Corresponding Author \\ Dr Shivani Gandhi \\ Post Graduate, Department of Pathology, Acharya Shri Chander College of Medical Science \& Hospital, \\ Sidhra, Jammu (J\&K), India \\ Email: dr.shivanigandhi@gmail.com, Phone: +91-9796651403

\begin{abstract}
Background: Liver is vulnerable to a wide variety of metabolic, toxic, circulatory insults. Microscopic examination of liver biopsy specimen remains the gold standard for the diagnosis of majority of the hepatic diseases.

Methods: A one year prospective study was conducted in the Post Graduate Department of Pathology, ASCOMS \& Hospital which included 72 liver biopsies. Each case was analysed with respect to age, gender and histological type.

Results: Out of the total 72 liver biopsy specimen, $86.11 \%$ cases were male, $14.28 \%$ were females. $33.33 \%$ cases were of hepatic tumors, $30.55 \%$ of cirrhosis, $11.11 \%$ and $9.72 \%$ of viral hepatitis and alcoholic hepatitis respectively. Hydatid cystic disease was seen in $2.7 \%$ cases and $5.55 \%$ cases showed granulomatous hepatitis.

Conclusion: Histomorphological study of liver biopsies help in evaluating the spectrum of various hepatic lesions.

Keywords: Histomorphological spectrum, hepatic lesions, liver biopsy.
\end{abstract}

\section{Introduction}

Liver has an essential task in maintaining the metabolic homeostasis of the body and regulates the metabolism of carbohydrates, lipid, vitamin and protein. It also regulates the synthesis of serum amino acids and detoxification of endogenous waste products. A wide range of primary and secondary diseases affect the liver. Among the primary diseases it most commonly involves toxic, metabolic, infectious and neoplastic insults. ${ }^{1}$ Light microscopic examination of the liver tissue remains an essential part of diagnostic procedure and follow up of the majority of liver diseases. ${ }^{2}$ Being a relatively safe 
and easy procedure, liver biopsy remains a vital tool in the diagnostic assessment and subsequent management of various liver diseases.

\section{Material and Methods}

The study was conducted in the Post Graduate Department of Pathology, ASCOMS and Hospital and consisted of prospective analysis of 72 liver biopsy specimens received in the department from February 2017 to February 2018. All relevant clinical details including liver function test and radiological findings were noted. Haematoxylin and eosin stained slides were examined under the microscope. Each case was analysed with respect to age, gender, clinical presentation and histological type. The findings were recorded and analysed.

\section{Results}

A total of 72 liver biopsy specimens were analysed as per age, gender and histology.

In this study, majority of the patients $86.11 \%$ were male $(n=62)$ and only $14.28 \%$ were females $(\mathrm{n}=10)$ (Table 1). The patient's ages ranged from 15 years to 85 years. Majority of the lesions were observed in the age group of 56-65 years followed by 66-75 years (Table 1 ).

The frequency of various hepatic lesions is shown in Table 2.

Majority of the hepatic lesions were tumours which accounted for $33.33 \%$ of the cases $(n=24)$. It was followed by cirrhosis seen in $30.55 \%$ cases $(n=22)$. Viral hepatitis and alcoholic hepatitis were observed in $11.11 \%$ and $9.72 \%$ cases respectively. Granulomatous hepatitis was observed in $5.55 \%$ cases $(n=4) .2 .7 \%$ cases showed hydatid cystic lesion of the liver.

Table 1: Basic demographics.

\begin{tabular}{|l|c|c|c|}
\hline \multirow{4}{*}{ Gender } & Category & $\begin{array}{c}\text { Number } \\
\text { of cases }\end{array}$ & $\begin{array}{c}\text { Percentage } \\
(\boldsymbol{\%})\end{array}$ \\
\cline { 2 - 4 } & Male & 62 & 86.11 \\
\cline { 2 - 4 } Age & Female & 10 & 14.28 \\
\cline { 2 - 4 } (years) & $15-25$ & 2 & 2.77 \\
\cline { 2 - 4 } & $26-35$ & 4 & 5.55 \\
\cline { 2 - 4 } & $36-45$ & 5 & 6.94 \\
\cline { 2 - 4 } & $46-55$ & 12 & 16.66 \\
\cline { 2 - 4 } & $56-65$ & 18 & 25 \\
\cline { 2 - 4 } & $66-75$ & 16 & 22.22 \\
\cline { 2 - 4 } & $>75$ & 15 & 20.83 \\
\hline
\end{tabular}

Table 2: Depicting the frequency of various hepatic lesions

\begin{tabular}{|l|c|c|}
\hline Hepatic lesions & Number of cases & Percentage (\%) \\
\hline Hepatic tumours & 24 & 33.33 \\
\hline Cirrhosis & 22 & 30.55 \\
\hline Viral hepatitis & 8 & 11.11 \\
\hline Alcoholic hepatitis & 7 & 9.72 \\
\hline Granulomatous hepatitis & 4 & 5.55 \\
\hline Hydatid cyst in liver & 2 & 2.7 \\
\hline Non specific & 5 & 6.94 \\
\hline Total & $\mathbf{7 2}$ & $\mathbf{1 0 0}$ \\
\hline
\end{tabular}

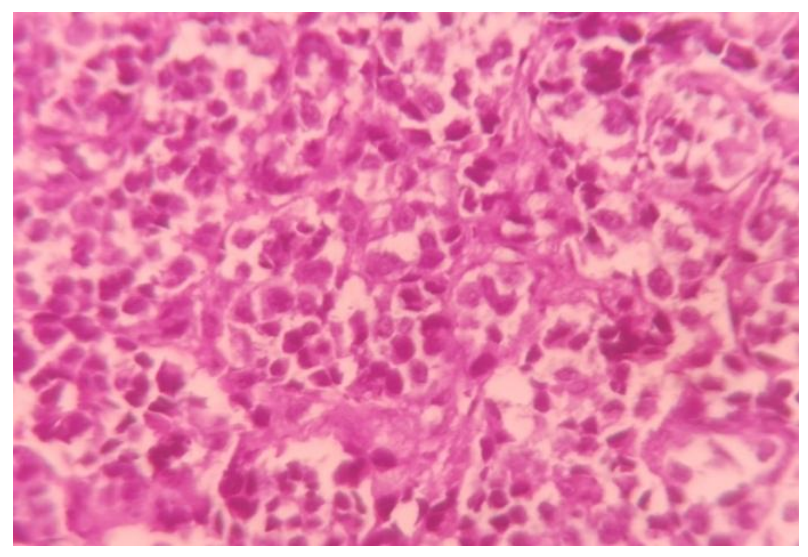

Fig 1: Photomicrograph showing the metastatic adenocarcinoma deposits in liver (H \& E, 40X)

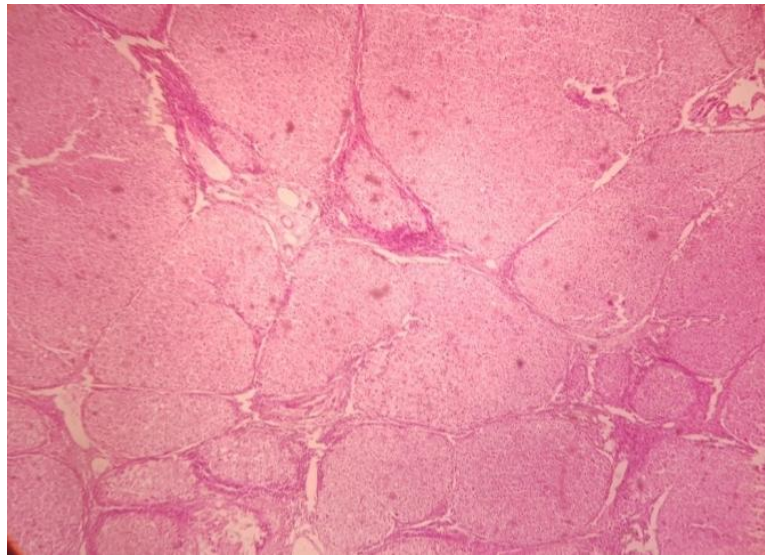

Fig 2: Photomicrograph of cirrhosis showing the nodules of variable sizes with thin fibrous septa (H \& E, 10X).

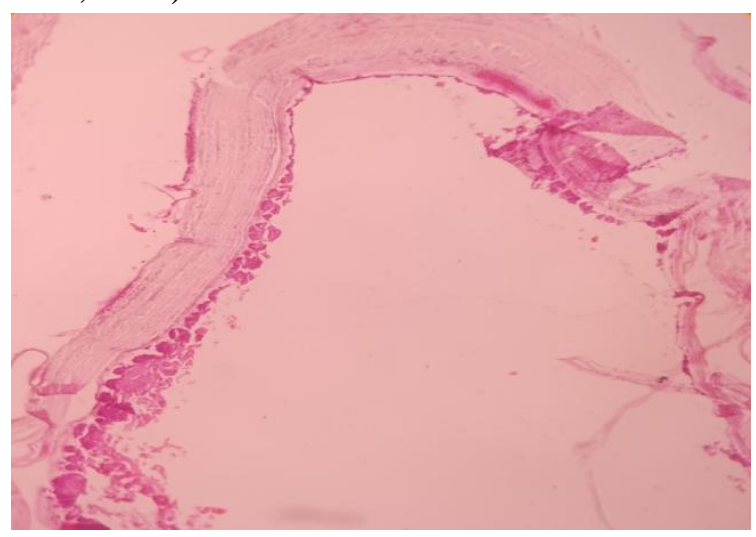

Fig 3: Photomicrograph showing cyst lined by laminated fibrous wall in hydatid cystic disease. 


\section{Discussion}

In the present study, 72 liver biopsy specimens were analysed. Majority of the hepatic lesions were found in the males $(n=62)$ whereas only $14.28 \%$ females were involved $(n=10)$. Becker et al, 1996 also found that majority of the hepatic lesions involved males. ${ }^{3}$ Our results were also consistent with the other studies. ${ }^{4}, 5,6$ However our results were different from the Indian study by Agrawal et al. ${ }^{7}$

In this study, the most commonly observed hepatic lesions were hepatic tumors and these results were in agreement with the other Indian study. ${ }^{4}$ Hepatocellular carcinoma was found to be the most common hepatic lesion with the clinical features of abdominal pain, ascites, hepatomegaly. There was the history of alcohol intake in $62.50 \%$ patients $(n=15) \& 4 \%$ cases showed the presence of HbsAg. These results were in concordance with the Indian study by Chawla and Sunila, 2013 and Agha et al, 2015.4, ${ }^{8}$ Microscopically, the lesion showed the tumour cells arranged in trabecular, solid, tubular, acinar pattern. The tumour cells are pleomorphic, polygonal with distinct cell borders, high N/C ratio, abundant granular eosinophilic cytoplasm, round nuclei with coarse chromatin and prominent nucleoli. Sinusoidal vessels were seen surrounding the tumour cells. Metastatic involvement of the liver was also seen in some of the cases $(n=5)$. The primary malignant tumour of gall bladder, pancreas, stomach, large colon, lung, breast often metastasises to the liver (Figure 1).

The next common hepatic lesion found in the present study was liver cirrhosis, seen in $30.55 \%$ of the cases (Figure 2). The various etiological agents that cause cirrhosis include alcoholic liver disease, hepatitis B infection, hepatitis C infection, Wilson disease, malnutrition, Primary biliary cirrhosis, Secondary biliary cirrhosis, hemochromotosis. Our results were similar with the other studies. ${ }^{4}, 7,9$ The results were also in concordance with the another study by MacSween and Scott, $1973 .{ }^{10}$

Viral hepatitis was found to be the third common hepatic lesion observed in $11.11 \%$ of the cases.
Majority of the cases showed the features of chronic hepatitis with the clinical presentation ranging from asymptomatic presentation to non specific features like fatigue, anorexia, hepatomegaly. Histologically, the lesion showed the presence of inflammatory infiltrate predominantly composed of lymphocytes and plasma cells with few neutrophils and macrophages in the portal triad and interface hepatitis. The results were comparable with the other studies. ${ }^{7}, 11$ Similar results were also observed in the study by Passarino et al, 2005. ${ }^{12}$ Hydatid cystic disease was found in $2.7 \%$ of the cases $(n=2)$. Hydatid disease is caused by the tapeworm Echinococcus granulosus with the majority of the cases occurring in the right hepatic lobe. Grossly, the lesion showed the presence of a solitary, unilocular cyst filled with clear fluid. Microscopically, the cyst showed the laminated avascular, eosinophilic membrane (Figure 3).

\section{Conclusion}

Liver biopsy is an essential, safe and reliable tool for the diagnosis, accurate assessment of severity and follow up of the various hepatic lesions. Histopathological examination of liver biopsy specimen remains a gold standard for the diagnosis and management of various liver disorders.

\section{References}

1. Pudale S.S, Ashok B.S, Ambadas P.G, Gajanan D.R, Pandharinath C.N. Study of liver pathology in autopsy cases- Original Article. Int J Curr Res. 2014; 6(3): 579597.

2. Desmet V.J., Rosai J. Liver. In: Rosai and Ackerman's surgical pathology. $10^{\text {th }} \mathrm{ed}$. Milan: Elsevier. 2012: 857-965.

3. Becker U, Deis A, Sorensen T.I.A., Gronbaek M, Johnsen KB, Muller CF et al. Prediction of Risk of Liver Disease by Alcohol Intake, Sex, and Age: A Prospective Population Study. Hepatology. 1996; 23(5): 1025-29. 
4. Chawla N and Sunila S. Spectrum of Histopathological Findings in Liver Biopsy. Indian Med Gaz. 2013: 363-67.

5. Abe H, Beninger P.R, Ikejiri N, Setoyama $\mathrm{H}$, Sata M, Tanikawa K. Light microscopic findings of liver biopsy specimens from patients with hepatitis type $\mathrm{A}$ and comparison with type B. Gastroenterology. 1982; 82: 938-47.

6. Ohnishi K., Iida S., Iwana S., Goto N., Nomur $\mathrm{F}$ et al. The effect of chronic habitual alcohol intake in the development of liver cirrhosis and Hepatocellular carcinoma in relation to hepatitis B surface antigen carriage. Cancer. 1982; 48: 67277.

7. Agrawal NS, Iqbal M.B, Patil AA, Karia KM, Kumar H. Study to Evaluate the Histopathological Spectrum of Hepatic Lesions in Liver Biopsies in a Tertiary Care Hospital. Annals of Pathology and Laboratory Medicine. 2018; 5(3): 228-33.

8. Agha A, Furnari M, Chakik RM, Ali MMA, Alsaudi D et al. Hepatocellular Carcinoma Is the Most Frequent Final Diagnosis of Focal Liver Lesions Identified in a Cross- Sectional Evaluation of Patients with Chronic Liver Disease in Saudi Arabia. Journal of Cancer Res. 2015.

9. Gall EA. Posthepatic, post necrotic and nutritional cirrhosis: A pathologic analysis. Am J Pathol. 1960; 36: 241-70.

10. MacSween RNM, Scott AR. Hepatic cirrhosis: A Clinicopathological review of 520 cases. J Clin Pathol. 1973; 26: 936-42.

11. Kringsholm B, Christoffersen P. Liver pathology in fatal drug addiction. Forensic Sci Int. 1982; 20(2): 141-51.

12. Passarino G, Ciccone G, Siragusa R, Tappero P, Mollo F. Histopathological findings in 851 autopsies, with toxicological and virological correlations. Am J Forensic Med Pathol. 2005; 26(2): 106-16. 